\title{
Sustainable pricing and revenue optimization for growing business and economies
}

\author{
Neha Gupta ${ }^{1}$ Mohini Agarwal ${ }^{1} \cdot$ Pratibha Garg $^{1} \cdot$ J. K. Sharma ${ }^{1}$ \\ Published online: 3 March 2021 \\ (c) The Author(s), under exclusive licence to Springer Nature Limited part of Springer Nature 2021
}

\section{Introduction}

For the growth of business and economies, decision making and sustainable practices play a significant role. Business that have employed optimization techniques in their practice are greatly benefitted in terms of pricing and revenue management. Accordingly, organizations are integrating sustainable business practices into their culture but still lacking in the adoption of sustainable pricing and revenue business models. In order to manage the decisions of pricing and revenue management in sustainable environment, the integration of knowledge, expertise, and implementation of various disciplines such as operations research, operations management, consumer behavior, business analytics, economics, software development, finance, marketing, and management science are required. From the viewpoint of researchers and practitioners, pricing and revenue management comprise of various activities pertaining to dynamic pricing, price drop, shelf space allocation, revenue prediction, purchase intention, buying behavior, profit optimization, market segmentation, analysis of financial indicators, capacity allocation, and buying decisions. This special issue aims to focus on these core areas of pricing and revenue management.

This special issue uncover the stimulus for innovative and sustainable business solutions concerning pricing, capacity management, business process management, and customer relation management with objective of profit and revenue

Neha Gupta

ngngupta4@gmail.com

Mohini Agarwal

mohini15oct@gmail.com

Pratibha Garg

pgarg1@amity.edu

J. K. Sharma

jksharma@amity.edu

1 Amity School of Business, Amity University, Noida, Uttar Pradesh 201313, India management. It has covered multiple disciplines such as management science, marketing, economics, information technology, analytics, and finance and accounting. This issue is based on qualitative, quantitative, and empirical research based papers from different viewpoint for pricing and revenue management. Moreover, designing specific conclusion is a challenge since the received papers pertain to different domains, but the selected papers contribute significantly in achieving the objectives of the special issue.

\section{Outline of the selected papers}

It is worth mentioning that price discrimination plays a significant role from a manufacturer perspective that leads to positive impact on the profitability of the chain members, selection of optimal price strategy, optimal sales volume, and optimal profit. Therefore, the first paper by Rofin and Mahanty proposed the price discrimination strategies by adopting two distinct channel power structures in their study. Firstly, a two-echelon Retailer-E-tailer DCSC model has been proposed to examine the competition between the retailer and e-tailer and to derive their optimal decisions under equal wholesale price strategy and discriminatory wholesale price strategy for the channel power structures considered. To assess the significance of the proposed model and to study the variations with respect to online channel preference of the product, a numerical illustration has been presented. It was found that it is optimal for the manufacturer to follow Discriminatory Wholesale Price Strategy irrespective of the channel power structure.

Globally, for the economic growth, the present focus is directed towards the attainment of sustainble goals and development under which energy sector plays a significant role. In view of the sustainable development, there has been a transition from non-renewable to renewable resources and in order to meet the growing requirement, there is the need to optimize. Hence, the second paper by Gupta et al. 
focused on revenue optimization. In this study, an MultiObjective Optimization Problem (MOOP) has been framed to determine the Renewable Energy Resources Mix. The proposed model has been examined under the fuzzy environment using Interactive fuzzy goal programming technique with three different functional forms of membership function namely linear, exponential, and hyperbolic. MOOP has been analyzed for the combination of three different types of solar panels and results depicted the supremacy of exponential and hyperbolic membership function over the linear.

Shelf Space Allocation (SSA) has always been an important aspect in the context of retail operation. The work by Shroff et al. has been focused on comparative study wherein the first case is based on the fact that retailer promotes the private brand manufactured by itself followed by the second case wherein retailer sells the private brand produced by another manufacturer. The manufacturer of the national brand acts as the Stackelberg given its power of setting wholesale prices. The results depicted that the manufacturer's profit reduces when they compete for space and make the retailer dominant when the latter is only involved in shelf space allocation without any in-house production.

Revenue management hold the central position in the tourism industry. The work by Joshi and Dhaigude focused on the capacity allocation for Homestay which can maximize the revenue during peak and slack season. Authors utilized TODIM and Monto-Carlo simulation with EMSR-b methods to understand the competitive positioning and booking limits determination. Their results convey that variety in available packages satisfies the customers and there is need to reduce protection interval for homestay owners.

Textile industry is one of the leading industry which creates pollution in India and to reduce the level of pollution; textile industry started focusing on sustainable apparel production. Therefore, in this area, Kumar and Mohan studied the sustainable factors that impact the green apparel purchase intention of educated millennial in India. In this study, TPB model has been extended and PLS-SEM utilized to confirm the proposed structural relationships.

Revenue generation is as important in retail electricity sector as in others, for which the dynamic pricing is one significant approach. The study by Mitra and Dutta focused on describing a novel method for segmenting the retail electricity market in a university setup. Cluster analysis and discriminant analysis have been used for classifying the customers into different segments and later the demand-price relationship and price elasticity have been examined. Appliance-specific consumption pricing is yet another method for enhancing demand response and promoting sensible consumption.
The understanding of financial indicators plays a significant role in revenue management of multi-sector companies. Therefore, the work by Hooda et al. presents the study for identification of the key financial indicators of high-performing companies with the help of an advanced multivariate discriminant function analysis through three group design. The results have implications for investors, management, credit rating agencies, mergers, and acquisitions, etc., as their decisions depend upon sound prediction and forecasting.

With the development and urbanization, various luxury brands are evolving day by day. This leads to complicate the buying decision making for customers. Therefore, Dangi et al. explored the antecedents of customer perception and its effect on the purchase intention first and then the impact on buying decision making about branded products especially luxury products. Also, the study explores the role of price discounts in converting intentions into buying decision. Authors used EFA, SEM, and Mediation methods for the analysis purpose and identified four major determinants. Also, they showed that the price discount plays a role of partial mediation, where due to price discount available for luxury branded products, the buying decision making has been reduced significantly.

A price drop is a typical limited time instrument utilized by brands and advertisers, to animate motivation purchasing just as to improve stationary sales. Therefore, Prakash et al. focused to study the effect of the price drop on the customer's perceived evaluation across selected product categories. Author firstly identified the top three product categories and then explored the factors of customers' perceived evaluation in case of a price drop using exploratory factor analysis. Finally, Multivariate Analysis of Variance has been utilized to check the effect of a price drop on identified perceived evaluation across selected product categories. Results depict that customers were more concerned about perceived quality in case of price drop in comparison to other identified factors.

Acknowledgements We would like to thank all reviewers for providing useful comments and the authors for contributing their research papers. Without your help, it would have been impossible to produce this special issue. We would also like to extent the gratitude to the Editor-in-Chief, Ian Yeoman, for his guidance and support in making this special issue possible.

Publisher's Note Springer Nature remains neutral with regard to jurisdictional claims in published maps and institutional affiliations. 